\title{
HYPERPLANE SECTIONS OF WEAKLY NORMAL VARIETIES IN POSITIVE CHARACTERISTIC
}

\author{
CATERINA CUMINO, SILVIO GRECO AND MIRELLA MANARESI
}

(Communicated by Louis J. Ratcliff, Jr.)

\begin{abstract}
It is known that the general hyperplane sections of a weakly normal projective algebraic variety are weakly normal if the ground field has characteristic zero. This is shown to be false in positive characteristic; counterexamples can be obtained by intersecting "bad" weakly normal varieties with suitable linear spaces.
\end{abstract}

\section{INTRODUCTION}

Weakly normal (WN) algebraic varieties were introduced by Andreotti and Bombieri in 1969, see [3]. A natural problem is to understand whether the general hyperplane sections of a WN subvariety of $\mathbf{P}^{n}$ are WN.

In characteristc zero this problem, studied in [1] and [2], was solved independently and affirmatively in [14] and [7]; a stronger statement also holds, see [8].

In positive characteristic an affirmative answer is known (see [7]) only for the class of those varieties which are $S_{2}$ and "WN1", i.e., WN and, roughly, well behaved in codimension 1 (see [9] and Definition 1 below).

In this paper we show (Theorem 1) that if any sufficiently general hyperplane section of an equidimensional WN variety $X \subseteq \mathbf{P}^{r}$ is WN1, then $X$ is also WN1. This easily implies that if $X$ is $\mathrm{WN}$ but not WN1 a suitable intersection of $X$ with a linear space (possibly the same $\mathbf{P}^{r}$ ) in $\mathrm{WN}$, but its general hyperplane sections are not such. And since such varieties do exist (see [9] and Appendix) it follows that the answer to the above question is negative in general.

Received by the editors May 2, 1988 and, in revised form August 1, 1988.

1980 Mathematics Subject Classification (1985 Revision). Primary 14M05; Secondary 14C20.

Key words and phrases. Weakly normal and/or WN1 algebraic varieties, general hyperplane section.

This paper was written with the financial support of M.P.I.

The authors are members of G.N.S.A.G.A. of the C.N.R. 
Our proof is algebraic, and is based on results from [9] and [6] concerning WN1 varieties and analytic branches, and makes use of Hironaka's theory of normal flatness, see [12].

\section{THE MAIN RESULT}

We work over an algebraically closed field $k$ and by (algebraic) variety we mean an algebraic $k$-scheme, i.e., a scheme of finite type over $k$. We recall the following definitions.

Definition 1 (see [3]). A reduced variety $X$ is said to be weakly normal (WN) if every birational morphism $X^{\prime} \rightarrow X$, which is also a universal homeomorphism, is indeed an isomorphism. (This is equivalent to say that $X$ coincides with its "weak normalization," see [3], Theorem 4.)

Definition 2 (see [9]). A variety $X$ is said to be WN1 if it is WN and moreover the normalization morphism $\bar{X} \rightarrow X$ is unramified in codimension 1 .

The main result in this paper is the following theorem, we shall prove in $\S 2$.

Theorem 1. Let $X \subseteq \mathbf{P}_{k}^{r}$ be a $\mathrm{WN}$ equidimensional projective variety of dimension $d \geq 2$. Let us assume that $X \cap H$ is $\mathrm{WN} 1$ for almost all hyperplanes $H \subseteq \mathbf{P}_{k}^{r}$.

Then $X$ is WN1.

(As usual "almost all hyperplanes" or "general hyperplane" means "all the hyperplanes corresponding to a suitable nonempty open subset of $\left(\mathbf{P}_{k}^{r}\right)^{*}$ ".)

Corollary 1. Let $X \subseteq \mathbf{P}_{k}^{r}$ be an equidimensional projective variety which is WN but not WN1. Then there is a linear subspace $L \subseteq P_{k}^{r}$ (possibly $L=\mathbf{P}_{k}^{r}$ ) such that:

(a) $Y=L \cap X$ is WN;

(b) a general hyperplane section of $Y$ is not $\mathrm{WN}$.

Proof. It is known that a WN curve is WN1 (see [9], (3.10)i). The conclusion follows easily by Theorem 1 .

Remark. As pointed out in the Introduction, Corollary 1 and the Appendix imply that there are $\mathrm{WN}$ integral varieties of dimension $0 \geq 2$ in some projective space whose general hyperplane sections are not WN. However from our method it is not clear if one can find counterexamples of any dimension.

\section{Proof of TheOREM 1}

In order to prove Theorem 1 we need a fact on associated graded rings (Lemma 1) whose geometric translation (Corollary 3) is well known, at least when $k=\mathbf{C}$. 
All rings in this paper are assumed to be commutative, with 1 and noetherian. We recall the following definition:

Definition 3. A prime ideal $\rho$ of a local ring $(R, m, k)$ is called admissible in $R$ if the local ring $R / \rho$ is regular and $\mathrm{Gr}_{\rho}(R)$ is a free $R / \mu$-module.

Lemma 1. Let $(R, m, k)$ be a local ring, let $\rho \subset R$ be an admissible prime ideal and let $x \in R$ be an element, whose image in $R / \rho$ belongs to a regular system of parameters.

Then there is an isomorphism of graded k-algebras $\operatorname{Gr}(R) \vec{\sim} \operatorname{Gr}(R / x R)[T]$ where $T$ is an indeterminate of degree 1 .

In particular $\operatorname{Gr}(R)$ and $\operatorname{Gr}(R / x R)$ have the same number of minimal primes. Proof. Let $r:=\operatorname{dim} R / \rho$ and let $x_{1}:=x, x_{2}, \ldots, x_{r}$ be elements of $R$, whose images $\bmod \rho$ are a regular system of parameters of $R / \rho$.

Let us consider the commutative diagram

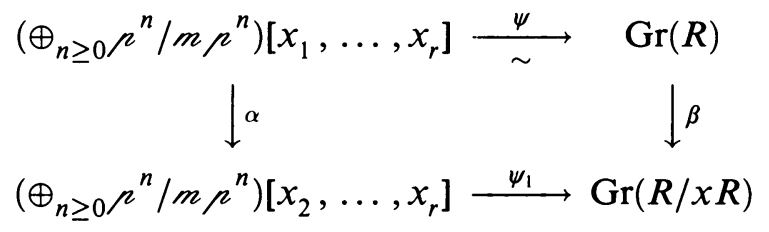

where $\psi$ is the Hironaka isomorphism defined by $\psi\left(x_{i}\right):=x_{i}^{*} \in \mathrm{m} / \mathrm{m}^{2}$ (see [12] Chap. II, Prop. 1, p. 183) and $\alpha$ is defined by $\alpha\left(x_{1}\right)=0, \alpha\left(x_{i}\right)=x_{i}$ for each $i=2, \ldots, r$. The map $\beta$ is induced by the canonical homomorphism $R \rightarrow R / x R$ and $\psi_{1}$ is induced by $\psi$, since $\beta(\psi(x))=\beta\left(x^{*}\right)=0$.

Since $\psi$ is an isomorphism, $x^{*}=\psi(x)$ is a nonzero divisor in $\operatorname{Gr}(R)$, hence $\operatorname{Gr}(R / x R) \simeq \operatorname{Gr}(R) /\left(x^{*}\right)$ (see [13] (1.1)); therefore $\psi_{1}$ is an isomorphism and the conclusion follows.

Corollary 2. Let the notation and the assumptions be as in Lemma 1. Then $R$ and $R / x R$ have the same multiplicity.

Proof. By Lemma 1 the Hilbert polynomial of $\operatorname{Gr}(R / x R)$ is the difference function of the Hilbert polynomial of $\operatorname{Gr}(R)$. The conclusion follows by definition of multiplicity.

Corollary 3. Let $X \subseteq \mathbf{P}_{k}^{r}$ be a variety; let $Y \subseteq X$ be a 1-codimensional irreducible subvariety.

Then there exists a nonempty open subset $U \subset Y$ such that for every closed point $x \in U$ and every general hyperplane $H$ through $x$ one has $T_{X, x} \simeq$ $T_{X \cap H, x} \times \mathbf{A}^{1}$ where $T_{X, x}$ stands for tangent cone of $X$ at $x$.

Proof. It follows by Lemma 1 and [12] Chap. II, Cor. p. 189.

Proof of Theorem 1. Let $Y \subseteq X$ be an irreducible subvariety of codimension 1 and multiplicity $s$. By [9] (2.1) and (2.3) we have to prove that there exists a nonempty open subset $U \subset Y$ such that $X$ has exactly $s$ distinct branches (which are linear by the multiplicity formula) at every closed point $x \in U$. 
Let $U \subset Y$ be a nonempty open subset such that:

(i) $X$ is admissible along $Y$ at every $x \in U$;

(ii) for every closed point $x \in U$ there exists a canonical surjective map \{branches of $X$ at $x\} \rightarrow \operatorname{Min}\left(\operatorname{Gr}\left(\mathscr{O}_{X, x}\right)\right)$;

(iii) the number of branches of $X$ at every closed point $x \in U$ is constant. Such a nonempty open subset $U$ of $Y$ exists by [12] (see Chap. II, Cor. p. 189), [6] (see (3.1)), [10] (see (1.6)); then by (i) and [12] the multiplicity at each closed $x \in U$ is $s$, and hence by (ii) and [10] (2.5) we have \# $\operatorname{Min}\left(\operatorname{Gr}\left(\mathscr{O}_{X, x}\right)\right) \leq$ \# \{branches at $x \leq s\}$ for all closed $x \in U$. Hence by (iii) it is sufficient to show that there is a closed point $z \in U$ such that $\# \operatorname{Min}\left(\operatorname{Gr}\left(\mathscr{O}_{X, z}\right)\right)=s$.

Let $H$ be a general hyperplane of $\mathbf{P}_{k}^{r}$. We have:

(a) $H \cap U \neq \varnothing$;

(b) $H \cap X=: X^{\prime}$ is WN1;

(c) every closed point of $U \cap X \subset X^{\prime}$ has multiplicity $s$ as a point of $X^{\prime}$ (see Cor. 2);

(d) there exists a nonembedded irreducible component $Z$ of $Y^{\prime}:=Y \cap H$ such that $Z \cap U \neq \varnothing$ (see [7] (3.4)).

By (b), (c), (d) and [9] (see (2.3)) there exists a closed nonsingular point $z \in Z \cap U$ such that

$$
\operatorname{Gr}\left(\mathscr{O}_{X^{\prime}, z}\right) \simeq k\left[x_{1}, \ldots, x_{s+d-1}\right] /\left(\ldots, x_{i} x_{j} \ldots\right)_{1 \leq i<j \leq s} .
$$

The conclusion follows by Lemma 1 applied to the local ring $R:=\mathscr{O}_{X, z}$, the prime ideal $\mu$ of $R$ associated to $Y$ and the local equation $t \in R$ of the hyperplane $H$.

\section{APPENDIX}

For sake of completeness we show that there are projective integral varieties which are WN but not WN1. The main idea of the construction is in [9], but we give a more complete and simpler account here.

Lemma 2. Let $A^{\prime}$ be a finitely generated integral normal $k$-algebra, $\mu \neq 0$ be a prime ideal of $A^{\prime}, R^{\prime}:=A^{\prime} / \mu, f: A^{\prime} \rightarrow R^{\prime}$ the natural homomorphism.

Let $R \subsetneq R^{\prime}$ be a $k$-subalgebra of $R^{\prime}$ such that $R^{\prime}$ is integral over $R$; let $K$, $K^{\prime}$ be the fractions fields of $R, R^{\prime}$, respectively, and assume $R=K \cap R^{\prime}$.

Let $A:=\left\{a \in A^{\prime} / f(a) \in R\right\}$. Then:

(i) $A$ is a finitely generated $k$-algebra with normalization $A^{\prime}$;

(ii) $A$ is $\mathrm{SN}$;

(iii) $A$ is WN if and only if $K=K_{\mathrm{rad}}$, where $K_{\mathrm{rad}}:=\left\{a \in K^{\prime} / a^{p^{p}} \in K\right.$ for some $e \geq 0\} \quad$ ( $p$ is the characteristic exponent of $k$ );

(iv) if furthermore ht $\mu=1, K=K_{\mathrm{rad}}$ and $K^{\prime} / K$ is inseparable, then $A$ is WN but not WN1. 
Proof. A direct computation shows that, since $R \subsetneq R^{\prime}$, the ideal $\rho$ is the conductor of $A$ in $A^{\prime}$; and since $\rho \neq 0$ the rings $A$ and $A^{\prime}$ have the same quotient field.

(i) Let $0 \neq x \in \rho$. Then $A^{\prime} \subset A x^{-1}$ and hence $A^{\prime}$ is integral over $A$ by [4] (5.1)(iv), so $A^{\prime}=\bar{A}$ by the above remark, since $A^{\prime}$ is normal. Moreover $A$ is a finitely generated $k$-algebra by [4] (7.8).

(ii) Let $t \in A^{\prime}$ be such that $t^{2}, t^{3} \in A$. Then $f(t)=f\left(t^{3}\right) / f\left(t^{2}\right) \in K \cap R^{\prime}=$ $R$, whence $t \in A$. Then $A$ is $\mathrm{SN}$ by [11].

(iii) Assume $K=K_{\mathrm{rad}}$. Let $t \in A^{\prime}$ be such that $t^{p} \in A$. Then $f(t)^{p} \in R=$ $R^{\prime} \cap K$, whence $f(t)^{p} \in K=K_{\mathrm{rad}}$, hence $f(t) \in K \cap R^{\prime}=R$, i.e., $t \in A$. Thus $A$ is WN by [15] Cor. p. 651.

Conversely assume that $A$ is WN and let $x \in K^{\prime}$ be such that $x^{p^{e}} \in K$. Since $R^{\prime}$ is integral over $R$, we have $x=a / s, a \in R^{\prime}, s \in R$. Then $a^{p^{e}} \in$ $R^{\prime} \cap K=R$. Put $a=f(t), t \in A^{\prime}$. Then $t^{p^{e}} \in A$, whence $t \in A$ by [15] Cor. p. 651. It follows that $a=f(t) \in R$ and hence $x \in K$. Then $K=K_{\text {rad }}$.

(iv) It follows by (iii) and [9] (1.1) and (1.2).

Corollary 4. There are projective integral varieties which are $\mathrm{WN}$ but not WN1. Proof. Let $k$ be an algebraically closed field of characteristic $p>2$, let $K:=$ $k(X, Y) \subset K^{\prime}=K(z):=K[Z] /\left(Z^{2 p}+X Z^{p}+Y\right)$. By [5], Chap. V, §9, Ex. 7, p. $136, K^{\prime} / K$ is a finite inseparable field extension and $K_{\mathrm{rad}}=K$.

Let $R:=k[X, Y]$ and $R^{\prime}:=k[X, Y, Z] /\left(Z^{2 p}+X Z^{p}+Y\right), A^{\prime}:=R^{\prime}[T]$, $\mu:=(T), f: A^{\prime} \rightarrow R^{\prime}=A^{\prime} / \rho$ the natural homomorphism. We observe that $A^{\prime}$ is regular and the quotient fields of $R$ and $R^{\prime}$ are $K$ and $K^{\prime}$, respectively.

By Lemma 2 the ring $A:=\left\{a \in A^{\prime} / f(a) \in R\right\}$ is a finitely generated WN but not WN1 $k$-algebra.

Let $\widetilde{V}$ be a projective closure of $V:=\operatorname{spec} A$ and let $W$ be the weak normalizaton of $\widetilde{V}$. Then $W$ is $\mathrm{WN}$ and contains an open subset isomorphic to $V$ (see [3]), hence it is not WN1. Moreover $W$ is projective by [7] and the conclusion follows.

Remark. From the proof of Corollary 4 we see tat there are WN varieties which are not WN1 of any dimension $\geq 3$ (add indeterminates if necessary). It is not clear to us whether one can have two-dimensional examples.

We observe that by the proof of Corollary 4 and Lemma 2 (iv) this is equivalent to the existence of field extensions $k \subset K \subset K^{\prime}$ as in Lemma 2 (iv), with $\operatorname{tr} . \operatorname{deg} K / k=1$.

\section{REFERENCES}

1. W. A. Adkins, A. Andreotti and J. V. Leahy, Weakly normal complex spaces, Contributi del C.L.I. di Sc. Mat. e Appl. n. 55, Accademia Naz. dei Lincei, Roma, 1981.

2. W. A. Adkins and J. V. Leahy, A topological criterion for local optimality of weakly normal complex spaces, Math. Ann. 243 (1979), 115-123.

3. A. Andreotti and E. Bombieri, Sugli omeomorfismi delle varietà algebriche, Ann. Scuola Norm. Sup. Pisa Cl. Sci. 23 (1969), 430-450. 
4. M. F. Atiyah and I. G. McDonald, Introduction to commutative algebra, Addison-Wesley P.C., Reading, 1969.

5. N. Bourbaki, Algèbre, Chap. V, Hermann, Paris, 1950.

6. C. Cumino, Tangent cones and analytic branches, Rev. Roumaine Math. Pures Appl. 31 (1986), 843-854.

7. C. Cumino, S. Greco and M. Manaresi, Bertini theorems for weak normality, Compositio Math. 48 (1983), 351-362.

8. C. Cumino, S. Greco and M. Manaresi, An axiomatic approach to the second theorem of Bertini, J. Algebra 98 (1986), 171-182.

9. C. Cumino and M. Manaresi, On the singularities of weakly normal varieties, Manuscripta Math. 33 (1981), 283-313.

10. S. Greco, On the theory of branches, Proc. Internat. Sympos Algebraic Geometry, Kyoto 1977, pp. 477-493.

11. E. Hamann, On the R-invariance of $R[x]$, J. Algebra 35 (1975), 1-17.

12. H. Hironaka, Resolution of singularities of an algebraic variety over a field of characteristic zero, Ann. of Math. 79 (1964), 109-326.

13. P. Valabrega and G. Valla, Form rings and regular sequenes, Nagoya Math. J. 72 (1978), 93-101.

14. M. Vitulli, The hyperplane sections of weakly normal varieties, Amer. J. Math. 105 (1983), 1357-1368.

15. H. Yanagihara, Some results on weakly normal rings extensions, J. Math. Soc. Japan 35 (4) (1983), 649-661.

Istituto di Matematica, facoltà di Scienze, Università, I-84100 Salerno, Italy

Dipartimento di Matematica, del Politecnico, Corso Duca degli Abruzzi, 24, I-10129 TORINO, ITALY 